\title{
Sobre los jóvenes: entre la multiplicidad, la diferencia y la incertidumbre
}

\begin{abstract}
A 1 mencionar la palabra adolescencia, ¿qué es lo primero que cada

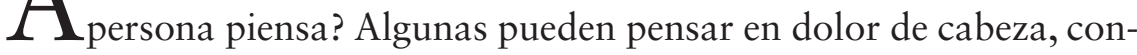
fusión, incertidumbre, otras pensarán en problemas, drogas, sexualidad, alcoholismo, miedo, otras tendrán ideas con respecto a desorden, anarquía, desafíos a la autoridad, suicidio y otras tal vez piensen en independencia, libertad, crecimiento, exploración, desarrollo del pensamiento, autonomía.
\end{abstract}

Hay muchas posturas e ideas con respecto a este momento vital en el que se toman decisiones, con o sin criterios claros, y que pueden afectar de manera significativa el resto de la vida. Estas decisiones van de la mano con los niveles de autonomía que comienzan a experimentar los jóvenes y que son naturales en su momento, pues alcanzan grados de libertad que en la niñez no tenían para tomar decisiones y vivir nuevas experiencias.

Al pensar en el contexto de la educación superior, los jóvenes que ingresan a la universidad se encuentran con nuevas experiencias de diferentes tipos y frente a las cuales deben tomar decisiones o asumir posturas haciendo uso de sus experiencias previas, de sus habilidades y destrezas, de sus recursos, de sus historias. Además, deben resolver nuevos conflictos, establecer tipos de relaciones diferentes a los que 
antes conocían y llevar consigo a este nivel de educación superior sus dilemas, preguntas, cuestionamientos, problemas, entre otros.

Por ello es necesario encontrar herramientas de apoyo para los jóvenes en las que puedan, de ser el caso, hallar solución a aquello que les aqueja, por medio de las cuales puedan expresar su sentir, pensar y actuar, entrar en dialogo consigo mismos y con otros sobre los dilemas que tienen. Construirse así como seres humanos desarrollados justamente en esa área: lo humano, para que de esta manera tomen decisiones que ayuden a su crecimiento y beneficio y se posicionen frente al mundo de una manera saludable a nivel mental, emocional, espiritual y conductual. Una de las herramientas posibles para el logro de estos objetivos es la arteterapia.

Anteriormente se abordaron conceptos e ideas importantes para entender qué es la arteterapia, ahora es necesario comprender al joven, entender su momento vital y lo que han detectado algunas universidades sobre lo que sucede con ellos a nivel personal, lo cual va más allá del conocimiento que la academia les pueda brindar. Es allí, en estos dilemas, dificultades y confusiones que tiene el joven, donde la arteterapia puede intervenir para apoyarle en su proceso de desarrollo personal.

\section{Algunas consideraciones sobre la adolescencia}

Con la llegada de la adolescencia se presentan múltiples cambios a nivel físico, según Papalia, Wendkos y Duskin (2010) hay un crecimiento de estatura, se presentan características sexuales, hay segregación de hormonas del mismo tipo, cambios en la voz, crecimiento de los músculos, entre otros. Los autores agregan que en este momento del ciclo vital es donde algunos jóvenes pueden presentar trastornos de la alimentación, de la imagen corporal, consumo y abuso de sustancias psicoactivas, se experimentan momentos y trastornos depresivos.

Estas características de los adolescentes son visibles en aquellos que conforman la población de esta investigación. Algunos de ellos las 
asumen de manera natural y se adaptan relativamente fácil a los cambios, a otros les cuesta más trabajo sentirse cómodos con su nuevo cuerpo y nuevas sensaciones. Se encuentran en el momento de conocer su sexualidad y tomar decisiones sobre su orientación, lo que puede resultar difícil y confuso para algunos de ellos; es momento de que sus ideas en torno al contacto con el sexo opuesto se materialicen y además definen la manera en que van a llevar a cabo sus prácticas sexuales.

En esta etapa se logra la última fase del desarrollo del pensamiento según Piaget (Papalia y otros, 2010), la etapa de las operaciones formales en la que el joven puede pensar de manera abstracta, comprender variaciones en el tiempo; no solo están ubicados en el aquí y el ahora, utilizan símbolos para representar otros símbolos, comprender y elaborar metáforas, imaginar posibilidades, elaborar hipótesis.

Estos cambios a nivel cognitivo implican cambios en el procesamiento de la información y por supuesto de la memoria. Papalia y otros autores (2010) mencionan que en esta etapa los lóbulos frontales del cerebro han madurado, aunque el nivel de desarrollo y la cantidad de conexiones neurales varía de persona a persona, por lo que es difícil unificar criterios para todos los adolescentes. Sin embargo, por lo general se presentan cambios estructurales a nivel cognitivo-neurológico, ya que la memoria de trabajo aumenta su capacidad, al igual que la memoria a largo plazo; la información que se almacena en este segundo tipo de memoria puede ser declarativa — saber qué-, procedimental — saber cómo- o conceptual —saber por qué-.

Otros cambios que se presentan son los funcionales, los cuales se relacionan con el aprendizaje, el recuerdo y el razonamiento. Según Kuhn (2006, citado en Papalia y otros, 2010), en la adolescencia hay un incremento continuo en la velocidad de procesamiento de la información y un mayor desarrollo de la función ejecutiva, que interviene con funciones como la atención selectiva, toma de decisiones, control inhibitorio de respuestas impulsivas y control de memoria de trabajo.

Se podría pensar entonces que en esta etapa el joven construirá un tipo de arte diferente y se expresará por medio de este de manera diferente. También podrá darle una interpretación a aquello que ha creado 
y elaborar conclusiones sobre su sentir y pensar con respecto al dilema que su obra le ha traído. La arteterapia es una forma de intervención que, a partir de la adolescencia, permite establecer significados y comprensiones sobre las situaciones que el joven que creó el arte, está atravesando, además, gracias a los procesos cognitivos del adolescente, puede generar hipótesis, ideas, creencias y comportamientos que le permitan sentirse mejor.

Además de estos cambios, es en la adolescencia cuando se experimenta por primera vez la búsqueda de identidad, la cual según Erickson (1950, citado en Papalia y otros, 2010) se entiende como una concepción coherente del yo compuesta por valores, metas y creencias con las que la persona adquiere un compromiso. Erickson menciona que este momento de búsqueda no necesariamente implica un malestar, sino que es parte de un proceso saludable y necesario para los seres humanos; el autor agrega que rara vez se resuelven los temas inherentes a la búsqueda de identidad en la adolescencia, ya que este dilema surge de nuevo y en varias ocasiones en la vida adulta.

Erikson (1950, citado en Papalia y otros, 2010) menciona que la identidad se construye en la medida en que los jóvenes resuelven tres temas importantes: elección de una ocupación, adopción de valores para su vida y el desarrollo de una identidad sexual satisfactoria. El autor menciona que la confusión con respecto a la identidad que se presenta en esta etapa es normal, sin embargo, se espera que el adolescente logre resolver los tres aspectos mencionados para tener cierta estabilidad, aunque más adelante, como ya se mencionó, puedan surgir de nuevo dudas frente a la identidad.

Con la arteterapia es posible apoyar al adolescente en la búsqueda de su identidad, pues la falta de resolución de ciertos temas puede generarle malestar. Además, la crisis de la identidad puede estar relacionada con una falta de autoconocimiento, proceso que se puede trabajar a partir de la arteterapia, logrando así niveles de equilibrio para que el adolescente pueda resolver sus dilemas, enfrentar situaciones, solucionar conflictos y encontrar su propio camino. 


\section{Riesgos psicosociales en la adolescencia}

La adolescencia, por ser un período crítico sometido a constantes cambios en el desarrollo físico, psíquico y social del adolescente, así como también en el marco familiar en que este se desenvuelve, se considera como un período de riesgo en el cual pueden darse las bases para la aparición de síntomas y enfermedades, así como también de alteraciones de la personalidad.

Debido al carácter de esta investigación, se considera importante hacer un análisis de los factores psicosociales de riesgo en la adolescencia. Para esto consideraremos como principales factores de riesgo psicológicos los siguientes, propuestos por Herrera (1999):

- Insatisfacción de las necesidades psicológicas básicas: dentro de ellas se pueden destacar la necesidad de autoafirmación, de independencia, de relación íntima personal y la aceptación por parte del grupo.

Patrones inadecuados de educación y crianza. Estos pueden ser:

- Sobreprotección: se puede manifestar de una manera ansiosa - al crear sentimientos de culpa en el adolescente- o de una manera autoritaria —al provocar rebeldía y desobediencia-.

- Autoritarismo: limita la necesidad de independencia del adolescente y mutila el libre desarrollo de su personalidad, para provocar como respuesta, en la mayoría de los casos, rebeldía y enfrentamientos con la figura de autoridad y pérdida de la comunicación con los padres.

- Agresión: tanto física como verbal, menoscaba la integridad del adolescente, su autoimagen y dificulta en gran medida la comunicación familiar. 
- Permisividad: esta tendencia educativa propicia la adopción de conductas inadecuadas en los adolescentes por carencia de límites claros.

- Autoridad dividida: este tipo de educación no permite claridad en las normas y reglas de comportamiento, y provoca la desmoralización de las figuras familiares responsables de su educación.

- Ambiente frustrante: cuando el adolescente no encuentra adecuadas manifestaciones de afecto, cuando hay censura inmotivada y frecuente hacia su persona, cuando se reciben constantes amenazas, castigos e intromisiones en su vida privada y cuando se aprecia un desentendimiento y alejamiento de las problemáticas que presenta.

- Sexualidad mal orientada: cuando por la presencia de prejuicios en relación con los temas sexuales, la comunicación en esta esfera queda restringida y el adolescente busca por otros medios, no siempre los idóneos, sus propias respuestas e informaciones, o en muchos casos mantiene grandes lagunas que le acarrean grandes problemas por el desconocimiento, la desinformación y la formación de juicios erróneos en relación con la sexualidad.

En relación con el riesgo social del adolescente se analizarán algunos factores sociales y ambientales que pueden conducir a resultados negativos en los jóvenes. Como principales factores de riesgo social Herrera (1999) propone:

- Inadecuado ambiente familiar: cuando la familia es disfuncional, no cumple sus funciones básicas y no están claras las reglas y los roles familiares se dificulta el libre y sano desarrollo de la personalidad del adolescente. Es necesario que exista un soporte familiar abierto, capaz de asimilar los cambios requeridos para la individualización del adolescente. 
- Pertenencia a grupos antisociales: este factor tiene como causa fundamental la satisfacción de la necesidad de autoafirmación y la necesidad del seguimiento del código grupal. Por lo general cuando los adolescentes no encuentran una vía adecuada de autoafirmación tratan de buscarla en este tipo de grupos donde fácilmente la encuentran, con el reconocimiento grupal ante la imitación de sus patrones inadecuados.

- La promiscuidad: es un factor de riesgo social que no solo puede ser motivo de embarazos precoces y enfermedades de transmisión sexual, sino que también propicia una autovaloración y una autoestima negativas que puede deformar la personalidad del adolescente.

- Abandono escolar y laboral: este hecho provoca que el adolescente se halle desvinculado de la sociedad y no encuentre la posibilidad de una autoafirmación positiva, al disminuir las posibilidades de comprobar sus destrezas para enfrentar los problemas y asumir responsabilidades, lo cual resquebraja su autoestima, la confianza en sí mismo y en sus posibilidades de desarrollo social.

- Bajo nivel escolar, cultural y económico: estos son elementos considerados como protectores del desarrollo y la salud; el hecho de presentar un déficit en ellos le impide al adolescente un enfrentamiento adecuado a las situaciones de conflicto.

De manera general se puede decir que el manejo de estos factores de riesgo permite identificar a aquellos adolescentes que están más expuestos a sufrir los daños que los aquejan, como accidentes, embarazos precoces, abuso de sustancias psicoactivas, enfermedades de transmisión sexual y suicidio; por lo cual se deben propiciar espacios en donde se promueva el desarrollo, crecimiento y maduración sana del adolescente, y en donde se le brinde posibilidades de enfrentar problemas con responsabilidad; además de oportunidades de autoafirmación positiva. 


\section{El joven universitario}

Es necesario además de revisar y entender al joven en su momento vital, entenderlo también en el contexto en el que se enmarca esta investigación: la universidad. El adolescente que ingresa y cursa sus estudios de pregrado tiene unas características particulares frente a las cuales es necesario prestar atención para poder definir áreas de intervención desde la arteterapia y entender de qué manera se le puede acompañar, con ayuda de la universidad, en su proceso de formación humana.

Alonso, Murcia, Murcia, Herrera, Gómez, Comas y Ariza (2007) realizaron una investigación sobre autoestima en estudiantes de primer semestre en una universidad de la costa colombiana y encontraron que la mayoría de estudiantes $-80 \%$ de la muestra - tienen niveles adecuados de autoestima, lo cual se evidenció por medio del autoconcepto, el autorrespeto y la autoaceptación. Este es un panorama alentador y fértil de trabajo, ya que da paso a la prevención, a la búsqueda de factores protectores, los cuales, según los autores de la investigación, son apremiantes trabajar desde el ingreso a la universidad.

González (2003, citado en Alonso y otros, 2007) realizó un estudio en el que pretendía revisar la autoestima en estudiantes universitarios y encontró, al igual que Alonso y otros, un buen nivel de autoestima en los estudiantes de primer semestre; sin embargo, al compararlo con estudiantes de semestres más avanzados, encontró que estos últimos tenían niveles más elevados de autoestima en comparación con los de primero. A esto se suma que el boletín informativo de la facultad de medicina de la Universidad de Chile (citado en Alonso y otros, 2007) menciona que los estudiantes que más toman el servicio de orientación psicológica que ofrece la universidad, son los de primero, tercero y quinto semestre. Los estudiantes de primero consultan debido al cambio que implica el ingreso a la vida universitaria, pues esto genera un choque que los lleva a pedir apoyo.

Para tener en cuenta, en los resultados de esta investigación sobre autoestima en estudiantes de primer semestre hay que considerar que, si bien los jóvenes tienen un buen nivel de autoestima, no se puede descuidar a aquel grupo de estudiantes que no la tienen. Si bien no son 
la mayoría, requieren de atención, sin descuidar aquellos que sí cuentan con buen nivel de autoestima pues son adolescentes que pueden potenciar habilidades para su futuro y colaborar en la continuidad de su desarrollo humano, en su autoconocimiento, autorregulación, autocompasión y ayudar a prevenir que en el futuro tomen decisiones que afecten de manera negativa su vida, como el consumo de sustancias, el embarazo adolescente, la deserción universitaria, entre otros.

Con la anterior idea se relaciona que la mayoría de adolescentes de la sociedad colombiana muestran una falta de respeto por sí mismos, lo que puede relacionarse con trastornos mentales como la depresión (Pardo, 2004, citado en Alonso y otros, 2007); además, una baja autoestima se relaciona con estructuras de la personalidad depresivas, narcisistas, timidez, ansiedad social y dificultades para relacionarse (Alonso y otros, 2007).

Para profundizar en el tema de la depresión, Arrivillaga, Cortez, Goicoechea y Lozano (2004) realizaron una caracterización de la depresión en jóvenes universitarios en una universidad en Cali y encontraron que entre el $25 \%$ y el $30 \%$ de la población presentaba algún nivel de depresión - leve, moderada y severa—, siendo más frecuente en mujeres y en personas de estratos socioeconómicos bajos. El $100 \%$ de los jóvenes con depresión severa manifestaron haber tenido intención de suicidiarse. Dentro de los niveles de depresión y en cuanto a los jóvenes que la presentaron, la mayoría de ellos mostraban síntomas de depresión leve, seguidos por los de depresión moderada y una minoría de casos se encontraban en depresión severa. También observaron una relación entre la depresión y el consumo de alcohol: a mayor nivel de depresión mayor consumo de la sustancia.

Arrivillaga y otros autores (2004) encontraron que algunos de los factores asociados a la depresión en la población de jóvenes universitarios, se asocian a la aparición de eventos críticos durante los seis meses anteriores al estudio, por ejemplo, inestabilidad económica, diagnóstico de enfermedad grave, muerte de un ser querido y separación de los padres. Las autoras mencionan que estas situaciones junto con un déficit de estrategias de afrontamiento apuntan al desencadenamiento de la depresión. Sue (1997, citado en Arrivillaga y otros, 2004) agrega que las personas que tienen una opinión pobre de sí mismas se juzgan 
constantemente o al mundo de manera negativa y, además, se dejan llevar por la presión; son personas que tienden a la depresión.

Si hay un sector de la población universitaria que presenta características o síntomas de depresión, es necesario trabajar con ellos y apoyarlos en su proceso de solución de la situación. No hay que identificarlos para etiquetarlos, aislarlos de los demás y tratarlos como enfermos mentales, sino por el contrario, ayudarles a explorar su malestar, a encontrar el sentido del mismo, a reconocer en sí mismos como este se manifiesta e ir orientando al joven en la solución de su situación y en el cambio de la posible versión negativa que tiene sobre sí mismo y sobre su entorno. Conectando así el sentido de vida que el joven ha construido, pues, como se comentaba anteriormente, es en la adolescencia cuando el joven comienza a preguntarse por su identidad y, con ello, por el sentido de su vida.

Magaña, Zabala, Ibarra, Gómez y Gómez (2004) mencionan que desde la visión humanista el vacío existencial es uno de los más graves problemas de nuestra sociedad, el cual se relaciona con varios de los problemas históricos que ha tenido la humanidad. Este vacío existencial puede enfrentarse por medio de la inteligencia, libertad, autonomía, capacidad de amar, responsabilidad creadora y poseedora de valores. Es de esta manera que cobra importancia trabajar en aquellas características que ayudan a que se vaya consolidando el sentido de vida. Magaña y otros (2004) realizaron una investigación con estudiantes de primer semestre y encontraron que la mayoría de estudiantes tienen un sentido de vida bien consolidado, por lo que se ven a sí mismos como capaces y responsables de sus propias vidas; sin embargo, advierten que no hay información sobre el sentido de vida de los estudiantes de semestres más avanzados, por lo que podrían presentarse cambios que son importante investigar y así conocer parte del desarrollo de los estudiantes a lo largo de su paso por la universidad.

Por otro lado, Magaña y otros autores (2004) encontraron que un $11.2 \%$ de estudiantes presentaron falta de sentido de vida, por lo que se hace necesario trabajar con esta población, ya que sucede la misma situación que en las investigaciones comentadas anteriormente. Si bien no es la mayoría, sí hay un grupo de adolescentes que requieren de acompañamiento, y la arteterapia es la oportunidad para trabajar 
situaciones como la búsqueda del sentido de vida sin sentirse señalados, acusados o diferentes a la mayoría.

Al tener en cuenta las características de los estudiantes universitarios hay un fenómeno que afecta a la comunidad en general y que preocupa a las universidades y a los gobiernos como foco de investigación para encontrar maneras de combatirlo: la deserción universitaria. Para poder entender este tema se parte de la pregunta sobre qué sucedió con el estudiante para que decidiera abandonar sus estudios, lo que sugiere que algo no anda bien y se deben implementar soluciones. Siendo así, ¿qué le sucede al estudiante para que deserte?

El Sistema para la Prevención de la Deserción de la Educación Superior (spadies) fue creado por el Ministerio de Educación Nacional (MEN) de Colombia para consolidar y ordenar información correspondiente a los estudiantes que han ingresado a las Instituciones de Educación Superior (IES) en Colombia, y así conocer los factores determinantes en la deserción, estimar el riesgo de deserción de los estudiantes y diseñar estrategias que apoyen al estudiante en su permanencia y graduación. La creación del spadies habla de una preocupación de gobierno por conocer a los estudiantes y acompañarlos para que logren la finalización de sus estudios, en un momento en que la deserción comenzó a preocupar a las universidades y al mismo gobierno.

Según este sistema, se han generado estrategias de apoyo económico y académico desde las IEs. Los esfuerzos, de acuerdo con las estadísticas presentadas en la página del SPADIES, se han concentrado en los apoyos financieros: para el 2014-2 un 20.16\% de los estudiantes contaron con este apoyo, mientras que un $6.57 \%$ de estudiantes tuvieron apoyo académico y un $3.76 \%$ apoyo de la IEs. En el sistema también se reporta que entre mayor número de apoyos tenga el joven, menor es la deserción; por ejemplo, para el décimo semestre $57.47 \%$ de los estudiantes que no recibieron ningún apoyo financiero por parte de la IEs desertaron, mientras que los que recibieron cuatro o más apoyos, desertaron en un $12.35 \%$; en cuanto a los apoyos académicos, de aquellos estudiantes que no recibieron ninguno desertaron el $52.18 \%$, mientras que los que recibieron cuatro o más apoyos desertaron en $23.43 \%$. Finalmente, desertaron $52.01 \%$ de los estudiantes 
que no recibieron ningún apoyo de la IEs o de otro tipo diferente a los anteriores en comparación con el $20.51 \%$ que desertaron habiendo recibido cuatro apoyos o más.

Es importante notar que los porcentajes de estudiantes que desertan cuando no reciben ningún apoyo se encuentran entre el $50 \%$ y el $60 \%$, y estos disminuyen notablemente en la medida en que se ofrece acompañamiento y ayuda al estudiante. Sin embargo, cuando se habla de otros tipos de apoyo de las IES no se especifica de qué tipo son, por lo que la información resulta insuficiente en este caso. Ahora bien, sí se puede pensar que en la medida en que se conoce al estudiante, se está cerca de él y se le acompaña de acuerdo con su necesidad, el nivel de deserción disminuye. Las estadísticas también muestran que para el décimo semestre la deserción total a nivel universitario es de alrededor del $40 \%$, la cual es una cifra muy alta e indica que poco más de la mitad de estudiantes que iniciaron la carrera la terminan.

González (2006) menciona que algunas causas de la deserción son de tipo externo, institucional, académico y personal; esta última tiene que ver con situaciones del estudiante, como sus motivaciones y actitudes, su condición económica, sus aspiraciones, la disonancia con sus expectativas, su insuficiente madurez emocional, el grado de satisfacción con la carrera, las expectativas de ingreso al mercado laboral, dificultades personales para adaptarse, integrarse con el ambiente universitario y falta de aptitudes o habilidades para la carrera escogida.

Frente a estos riesgos de deserción que presenta el estudiante, González (2006) propone algunas estrategias para trabajar con ellos y así disminuir los índices de abandono. Entre los elementos que propone, menciona que es importante ayudar al estudiante con su autoestima y autoconocimiento, trabajar la motivación y la autodeterminación, ayudarles a aprender a manejar su ansiedad, fortalecer las metodologías de resolución de conflictos, además de otros elementos relacionados con lo institucional, lo académico y los estilos y los procesos de aprendizaje. Estos elementos hacen alusión a la dimensión humana y al desarrollo personal, los cuales son necesario atender en la universidad no solo para prevenir la deserción, sino para formar seres humanos, objetivo superior en la formación de profesionales. 
En su investigación sobre bienestar psicológico en estudiantes universitarios, Salanova, Martínez, Bresó, Llorens y Grau (2005) encontraron una relación significativa entre buen rendimiento académico y bienestar psicológico: a mayor rendimiento menor agotamiento, mayor eficacia en el estudio, mayor vigor, mayor dedicación, mayor autoeficacia, mayor satisfacción y mayor felicidad asociada con el estudio, además hay bajos niveles de propensión al abandono. Se podría pensar entonces que el estudiante que experimenta bienestar psicológico ofrece mejores resultados y tiene pocas posibilidades de abandonar sus estudios, por lo que habría que ayudarlo a mantener o mejorar su bienestar — de manera que no solo tenga mejores calificaciones, sino que también se sienta mejor consigo mismo y con su ambiente-, acudir a factores protectores en el estudiante y promoverlos en el ambiente académico, además de fomentar las capacidades resilientes en ellos para afrontar adversidades.

Es necesario aclarar cuáles serían esos factores protectores por trabajar en los adolescentes. Werner (1982, citada en Peralta, Ramírez y Castaño, 2006) propuso los siguientes después de trabajar con niños que se habían enfrentado a situaciones de riesgo psicosocial. Son factores que, al promoverlos en los estudiantes, pueden ayudarlos a enfrentar situaciones y resolver problemas: autoestima, introspección, independencia, capacidad de relacionarse, iniciativa, moralidad, humor y pensamiento crítico. A estos aspectos los denominó pilares de la resiliencia, ya que ayudaron a los niños con los que investigó a convertirse en adultos competentes, seguros y afectuosos.

Por otra parte, Wolin y Wolin (1993) utilizan la teoría del mandala de la resiliencia para trabajar otros factores protectores, aunque retoman varios de los propuestos por Werner:

Introspección: refiere a la capacidad de comprender las cualidades de sí mismo y de los otros, y poder reflexionar de una forma activa y personal sobre las experiencias vividas.

Interacción: es la habilidad para establecer lazos íntimos y satisfactorios con otras personas, a fin de balancear la propia necesidad de empatía y aptitud para brindarse a otros. 
Iniciativa: es el placer de exigirse y ponerse a prueba en tareas progresivamente más exigentes; se refiere a la capacidad de hacerse cargo de los problemas y de ejercer control sobre ellos.

Independencia: es la capacidad de establecer límites entre uno mismo y los ambientes adversos; alude a la capacidad de mantener una sana distancia emocional y física, sin llegar al aislamiento.

Humor: es la capacidad de encontrar lo cómico en la tragedia; se mezclan el absurdo y el horror en lo risible de esta combinación.

Creatividad: significa crear e innovar en lo personal, lo familiar, lo artístico, lo científico y lo social para transformar la realidad por medio de la solución de problemas.

Moralidad: es el deseo de una vida personal satisfactoria, amplia y con riqueza interior; se refiere a la conciencia moral, a la capacidad de comprometerse con valores y de discriminar entre lo bueno y lo malo (Peralta, Ramírez y Castaño, 2006, p. 199).

Comprendiendo un poco los dilemas de los jóvenes universitarios, sus características vitales, revisando su situación al ingresar a la universidad y entendiendo los puntos humanos en los que se les puede apoyar, se puede comprender la necesidad y la importancia de cátedras que acompañen su desarrollo emocional y personal con estrategias como la arteterapia, que colaboran en la resolución de problemas y la potenciación de sus recursos, lo cual tiene un impacto no solo en el joven, sino en el contexto en el que habita y habitará. 